Communication

\title{
Evaluation of a Simplified Method for GC/MS Qualitative Analysis of Polycyclic Aromatic Hydrocarbons, Polychlorinated Biphenyls, and Organic Pesticides Using PARADISe Computer Program
}

\author{
Łukasz Dąbrowski (iD) \\ Department of Food Analysis and Environmental Protection, Faculty of Chemical Technology and Engineering, \\ UTP University of Science and Technology, 3 Seminaryjna Street, 85-326 Bydgoszcz, Poland; lukas@utp.edu.pl; \\ Tel.: +48-523-749-014
}

Received: 1 August 2020; Accepted: 14 August 2020; Published: 15 August 2020

\begin{abstract}
For complex matrices such as environmental samples, there is usually a problem with not fully resolved peaks during GC/MS analysis. The PARADISe computer program (based on the PARFAC2 model) allows the identification of peaks using the deconvoluted mass spectra and the NIST MS library. The number of repetitions required by this software (at least five) is a real limitation for the determination of semi-volatile compounds, like polycyclic aromatic hydrocarbons, polychlorinated biphenyls, and organic pesticides in environmental samples. In this work, the method to overcome this condition was proposed and evaluated. The sets of the five files required by PARADISe were prepared by mathematically modifying the original GC/MS chromatograms obtained for the standard mixture (C $=2 \mu \mathrm{g} / \mathrm{mL}$ of 40 compounds) and real sample extracts (soil samples with different total organic carbon content and one cardboard extract) spiked with standards. Total average match factor for all the substances identified in a standard mixture was 874 (near 900-“excellent match"), and for all the substances in the real samples, it was 786 (near 800- "good match"). The results from PARADISe were comparable to those obtained with other programs: AMDIS (NIST) and MassHunter (Agilent), tested also in this work. PARADISe software can be effectively used for chromatogram deconvolution and substance identification.
\end{abstract}

Keywords: PARADISe software; data processing; mass spectra deconvolution; GC/MS

\section{Introduction}

Environmental samples are usually a mixture of many different substances. The main problem of substance identification is the coelution of several not fully separated compounds. In this case, there are several options for improving the selectivity of the final analysis method. One of them is the confirmation of GC/MS data by parallel analysis of the sample with a selective detector [1,2], which provides complementary information on the structure of the compound.

In the case of lack of these additional data, qualitative analysis is usually based on GC/MS results only. It is performed using various deconvolution algorithms. Comparative studies of deconvolution computer programs and algorithms have already been described in many papers [3-7]. Among the various programs, AMDIS (automated mass spectral deconvolution and identification system) from NIST serves as a reference for its popularity and free access. Reliability of the results generating by this program (i.e., the effectiveness of identification) depends on the number of operational settings (e.g., deconvolution settings) of the software and GC $[3,8,9]$. It is known from various experiments that 
AMDIS tends to produce many false-positive components $[3,10]$. One of the AMDIS-based software is-commercially available from Agilent-MassHunter (MH) [11]. It allows to find compounds by chromatogram deconvolution, and then identify them by searching MS libraries. It is relatively easy, fast and accurate-without the user having to experiment with the operating settings [12].

Another approach to GC/MS data deconvolution is based on a model called PARAFAC2 (PARAllel FACtor analysis 2) [13-16]. This model allows the mass spectrum to be extracted for each component by combining information from multiple chromatograms obtained for the same sample. The background can be modelled and separated as one or more components. PARAFAC2 usage is limited to the mathematical users and needs extensive coding [11] (usually in MATLAB). Nevertheless, it has been used in many applications due to its high efficiency of chromatogram deconvolution. It was used, inter alia, to identify and quantify compounds in wine and tobacco [17], volatile organic compound emissions from poultry farm [18], compounds which permeate through the membrane from industrial dairy ingredient production used as process water [19] and others [6,20].

The graphical user interface for PARAFAC2 was developed at the University of Copenhagen and is called PARADISe (PARAFAC2 based Deconvolution and Identification System) [5,11]. Johnsen et al. (2017) characterized the PARADISe system's limitations and advantages [11]. This computer program is becoming more and more popular recently. It was used for the non-target analysis of volatile compounds in olive oil [21], wood smoke volatile composition [22], products from pyrolysis of poplars [23], and composition of cashew apple juice [24].

One of the requirements of the PARADISe system is that "at least five samples with independent variations must be included in the sample set" [11]. It is a real limitation in semi-volatile environmental pollutants determination (like polycyclic aromatic hydrocarbons: PAHs, polychlorinated biphenyls: PCBs, organic pesticides) to obtain more than several replicates of the sample. Soil, sediment, river water, and other samples contain — even after a thorough cleaning-many different substances that can contaminate the GC/MS system. This leads to progressive deterioration in instrument performance caused by the ion source and optics contamination [12]. Therefore, identification of non-targeted compounds involves a rough analysis of the sample extract, usually based on one to three samples.

In order to be able to use PARADISe in such a case, a simplified method of analyzing the GC/MS data has been proposed, based on a limited set of data derived from one chromatogram. Additional chromatograms were generated by mathematical modifications (shifting the peak retention time and multiplying the signal to simulate "independent variations" of the samples) of the original one to meet the requirements of the software. The aim of this work was to evaluate the usage of PARADISe for the analysis of polycyclic aromatic hydrocarbons, polychlorinated biphenyls, and organic pesticides. The results achieved in this way were compared to the results obtained from AMDIS (NIST) and MassHunter (MH) by Agilent.

\section{Results and Discussion}

Sample chromatograms obtained from the GC/MS analysis of the standard mixture and real samples are shown in Figure 1.

\subsection{Standard Mixture Analysis}

The performance of the PARADISe software was evaluated using standard analyte solutions at the concentration of $2 \mu \mathrm{g} / \mathrm{mL}$. Mixtures of lower concentrations $(1.3 \mu \mathrm{g} / \mathrm{mL}, 0.75 \mu \mathrm{g} / \mathrm{mL}$, and lower), were tested, but many analytes were not found (mainly PAHs and organophosphorus pesticides). A similar trend was also observed when using other deconvolution packages (AMDIS, MassHunter). Further experiments were carried out at the concentration of the standard mixture of $2 \mu \mathrm{g} / \mathrm{mL}$. 


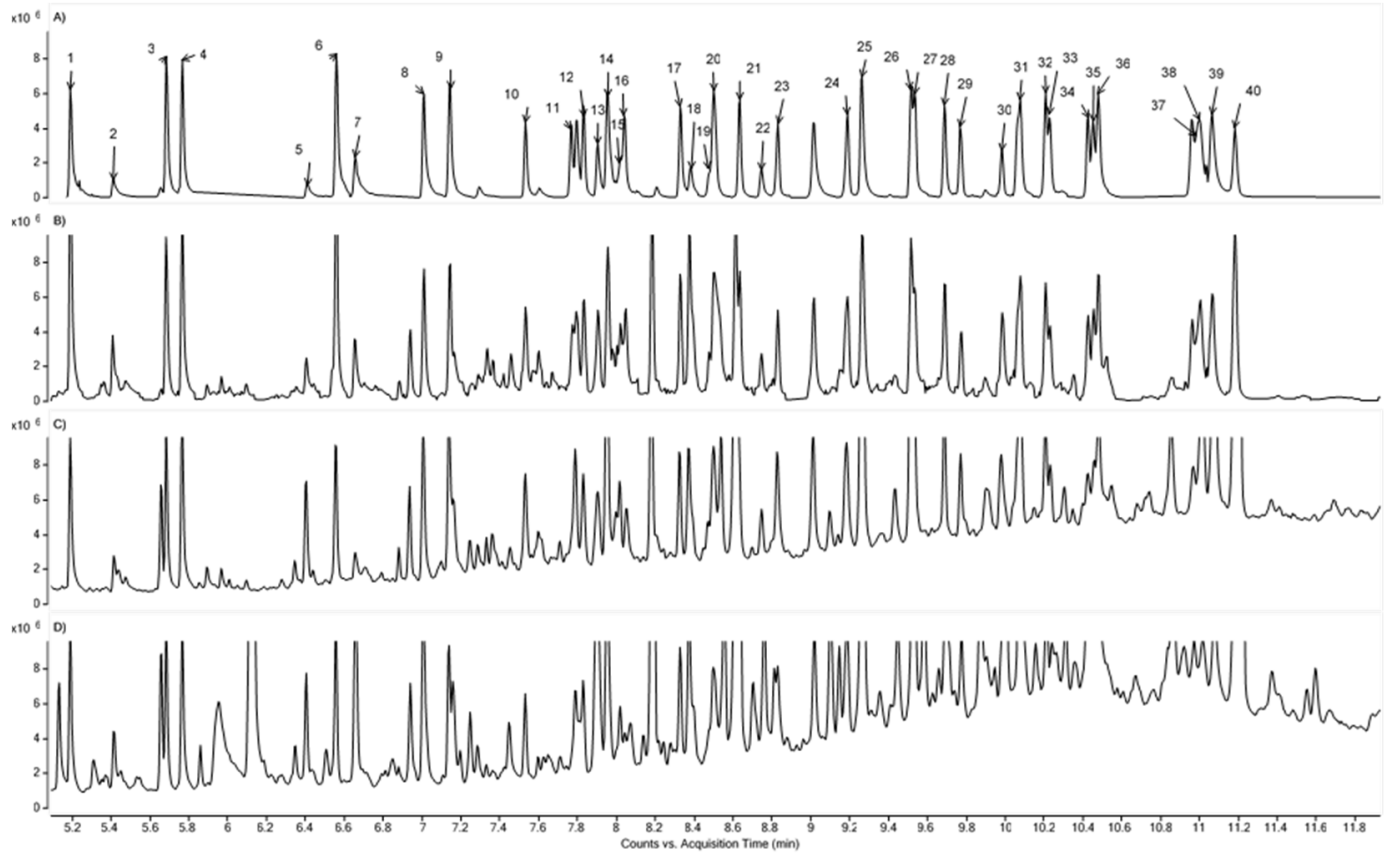

Figure 1. Sample chromatograms of (A) the standard mixture $(2 \mu \mathrm{g} / \mathrm{mL})$ and extracts from the samples: (B) S1, (C) S2, (D) S3 with added standards at $2 \mu \mathrm{g} / \mathrm{mL}$. The labels above the peaks on the chromatogram (A) correspond to the No column in Table 1.

The results obtained from the PARADISe program were presented in Table 1 (the number of repetitions $=3$, which is typical for this type of experiments [3,4]). All analytes were correctly identified using PARADISe program.

Due to the similar structure, it was not possible to distinguish some compounds (in any of the tested programs), i.e., isomers of $\mathrm{HCHs}$, $\mathrm{PCB}$ congeners with the same number of chlorines, 1-methylnaphthalene and 2-methylnaphthalene or PAHs with the same formula. In this study findings of the compound with the same formula were treated as a positive hit.

Average mass factors (AMFs) and average reversed match factors (ARMFs) were calculated by taking the match factors from all three repetitions of the analysis (Table 1). For fenchlorphos the average match factor is below 700 - probably because of the high similarity of its mass spectrum to co-eluting heptachlor (which MS spectrum is more complicated and consists of different ions, partially the same as fenchlorphos). There was also a problem in chrysene detection-only one positive hit. For all other compounds, PARADISe correctly detected them in three replicate analysis with a high match factor, even if the substances were not fully separated (e.g., 4,4'-DDT, endosulfan sulfate, and PCB 137) and their resolution factor was close to zero. According to the NIST MS Library User's Guide, match factor (MF) can be classified in the following way: "900 or greater is an excellent match; 800-900, a good match; 700-800, a fair match and less than 600 is a very poor match" [25]. The results should also be in agreement for two or more independent analyses [4]. The most numerous group of the results (85\%) contains those with AMF above 800. There is no case where AMF is less than 600. Similar criteria, as described above, were applied to the results obtained with the AMDIS software.

For $\mathrm{MH}$, another algorithm for calculating the quality of the spectral match is used, called Score. The result was considered positive if the Score was higher than $70 \%$. By analyzing the results obtained with MassHunter, two compounds were detected with low probability, i.e., anthracene (1 hit) and methoxychlor (MH Score for this compound below 70, Table 1). Problems with the correct identification of these two compounds also occurred when using AMDIS: 2 positive hits (MF > 700). 
Table 1. Average NIST MS search match factor (AMF), average NIST MS search reversed match factor (ARMF), and the number of replicates for the standard mixture (std), sample S1 (S1), sample S2 (S2), and sample S3 (S3) for PARADISe computer program, MassHunter (MH), and AMDIS software.

\begin{tabular}{|c|c|c|c|c|c|c|c|c|c|c|c|c|c|c|c|c|c|c|}
\hline \multirow[b]{3}{*}{ No } & \multirow[b]{3}{*}{ Compound } & \multirow{3}{*}{$\begin{array}{c}\text { RT } \\
\text { [min] }\end{array}$} & \multicolumn{6}{|c|}{ Standard Mixture } & \multicolumn{10}{|c|}{ Real Samples } \\
\hline & & & \multicolumn{3}{|c|}{ PARADISe } & \multirow{2}{*}{$\begin{array}{l}\text { MH } \\
\text { std }\end{array}$} & \multicolumn{2}{|c|}{ AMDIS } & \multicolumn{3}{|c|}{ PARADISe } & \multirow[b]{2}{*}{ S3 } & \multicolumn{3}{|c|}{ MH } & \multicolumn{3}{|c|}{ AMDIS } \\
\hline & & & AMF & ARMF & std & & std & AMF & ARMF & S1 & S2 & & S1 & S2 & S3 & S1 & S2 & S3 \\
\hline 2 & Dichlorvos & 5.41 & 835 & 884 & 3 & 3 & 3 & 796 & 826 & 2 & 3 & 3 & 3 & 3 & 3 & 3 & 3 & 3 \\
\hline 3 & 2-methylnaphthalene & 5.68 & 867 & 900 & 3 & 3 & 3 & 893 & 928 & 3 & 3 & 3 & 3 & 3 & 3 & 3 & 3 & 3 \\
\hline 4 & 1-methylnaphthalene & 5.77 & 922 & 931 & 3 & 3 & 3 & 903 & 924 & 3 & 3 & 2 & 3 & 3 & 3 & 3 & 3 & 3 \\
\hline 7 & o-hydroxybiphenyl & 6.70 & 888 & 914 & 3 & 3 & 3 & 888 & 905 & 3 & 3 & 3 & 3 & 3 & 3 & 3 & 3 & 3 \\
\hline 8 & Fluorene & 7.01 & 890 & 929 & 3 & 3 & 3 & 888 & 907 & 2 & 3 & 3 & 3 & 3 & 3 & 3 & 3 & 3 \\
\hline 9 & РCB 10 & 7.14 & 822 & 887 & 3 & 3 & 3 & 844 & 904 & 3 & 3 & 3 & 3 & 3 & 3 & 3 & 3 & 3 \\
\hline 10 & $\mathrm{a}-\mathrm{BHC}$ & 7.53 & 948 & 965 & 3 & 3 & 3 & 872 & 907 & 3 & 3 & 3 & 3 & 3 & 3 & 3 & 3 & 3 \\
\hline 11 & b-BHC & 7.76 & 923 & 950 & 3 & 3 & 3 & 698 & 776 & 3 & 3 & 0 & 2 & 1 & 0 & 3 & 3 & 1 \\
\hline 12 & Lindane & 7.83 & 920 & 938 & 3 & 3 & 3 & 848 & 889 & 3 & 3 & 2 & 3 & 3 & 3 & 3 & 3 & 3 \\
\hline 13 & Pyrimethanil & 7.92 & 793 & 954 & 2 & 3 & 3 & 701 & 810 & 2 & 3 & 3 & 3 & 3 & 1 & 3 & 3 & 3 \\
\hline 18 & Parathion-methyl & 8.39 & 761 & 842 & 3 & 3 & 3 & 488 & 736 & 1 & 0 & 0 & 2 & 1 & 3 & 3 & 3 & 3 \\
\hline 19 & Heptachlor & 8.50 & 724 & 728 & 3 & 3 & 3 & 683 & 809 & 3 & 1 & 3 & 3 & 3 & 3 & 3 & 3 & 3 \\
\hline 20 & Fenchlorphos & 8.51 & 664 & 728 & 2 & 3 & 3 & 629 & 703 & 3 & 3 & 2 & 3 & 3 & 3 & 3 & 3 & 3 \\
\hline 21 & PCB 52 & 8.63 & 944 & 962 & 3 & 3 & 3 & 857 & 877 & 3 & 3 & 0 & 3 & 3 & 0 & 3 & 3 & 2 \\
\hline 22 & Chlorpyrifos & 8.80 & 900 & 906 & 3 & 3 & 2 & 474 & 467 & 3 & 3 & 0 & 1 & 0 & 0 & 0 & 0 & 0 \\
\hline 23 & Aldrin & 8.83 & 919 & 921 & 3 & 3 & 3 & 745 & 739 & 3 & 3 & 3 & 3 & 3 & 3 & 3 & 3 & 3 \\
\hline 24 & Heptachlor epoxide & 9.19 & 887 & 890 & 3 & 3 & 3 & 741 & 792 & 3 & 3 & 3 & 3 & 3 & 3 & 3 & 3 & 3 \\
\hline 25 & Fluoranthene & 9.26 & 875 & 938 & 3 & 3 & 3 & 871 & 924 & 3 & 3 & 3 & 3 & 3 & 3 & 3 & 3 & 3 \\
\hline 26 & Pyrene & 9.52 & 857 & 925 & 3 & 3 & 3 & 825 & 911 & 3 & 3 & 3 & 3 & 3 & 3 & 3 & 3 & 3 \\
\hline 27 & a-endosulfan & 9.53 & 926 & 929 & 3 & 3 & 3 & 775 & 831 & 3 & 0 & 1 & 3 & 3 & 3 & 3 & 3 & 3 \\
\hline 28 & $4,4^{\prime}-\mathrm{DDE}$ & 9.69 & 948 & 975 & 3 & 3 & 3 & 858 & 914 & 3 & 3 & 3 & 3 & 3 & 3 & 3 & 3 & 3 \\
\hline 29 & Dieldrin & 9.77 & 930 & 931 & 3 & 3 & 3 & 810 & 830 & 3 & 2 & 3 & 3 & 3 & 3 & 3 & 3 & 3 \\
\hline 39 & Chrysene & 11.10 & 881 & 921 & 1 & 3 & 3 & 903 & 933 & 3 & 3 & 3 & 3 & 3 & 3 & 3 & 3 & 2 \\
\hline 40 & PCB 180 & 11.18 & 944 & 962 & 2 & 3 & 3 & 747 & 854 & 3 & 1 & 1 & 0 & 2 & 2 & 2 & 3 & 3 \\
\hline
\end{tabular}

\subsection{Real Sample Analysis}

Sample chromatograms of the extracts from the samples S1, S2, S3 (with added standards at $2 \mu \mathrm{g} / \mathrm{mL}$ ) were presented in Figure 1B-D, respectively. Additional peaks from the sample components and the increase of the baseline level can be observed on chromatograms. Compared to the standard chromatogram of the mixture, an increase in the height of the analyte peaks was also noted. This may be due to coelution with substances present in the extracts, as well as matrix-induced signal enhancement phenomena [26]. For comparison, all chromatograms are on the same OY scale.

The results obtained from PARADISe program are given in Table 1. Average match factors (AMFs and ARMFs) were calculated from the MFs obtained for all replicates of three different real samples. Additionally, the number of positive identifications in three replicate analysis were presented (similarly to the results for standard mixture). Identification of the analytes in more complex mixture leads to reduce detection efficiency in the case of higher organic carbon content (samples S2, S3). Coelution of analytes with large amounts of phthalates, siloxanes (e.g., from pipette tips, column bleed, and other sources) and other substances present in the sample can be observed in real samples. In such cases, the analytes were not identified, due to the overloaded peaks, which should be considered as one component in the PARAFAC2 model [27]. As an example: PCB 180 has very similar retention time (11.18 $\mathrm{min}$ ) to bis(2-ethylhexyl) phthalate (identified with MF = 805), which creates an overloaded peak. As a result, PCB 180 is often not detected. After diluting the extract fivefold, re-analyzing the 
sample with GC/MS and increasing the number of components in the model to eight, PCB 180 was identified, but with an MF of only 550 (RMF $=720$ ). In this case, this result should be confirmed by another method of identification.

In the case of two analytes: anthracene and endrin, the AMF is 642 . This is below 700 and can be classified as almost as a "fair match" [25]. Average reversed MF for anthracene is quite high and equals 829. There is a problem with the detection of two other compounds: parathion-methyl, chlorpyriphos (AMF: 488 and 474, respectively). This is due to the fact that huge amounts of substances originated from the real samples can be observed on chromatograms at retention times similar to the analytes (Figure 1B-D). Chlorpyriphos was also not detected either with MH (only 1 hit) or AMDIS (no hits). Analyzing the results in Table 1 it can be concluded that PARADISe software provides complementary information to MH or AMDIS. There are cases where PARADISe detects compounds in a more effective way than $\mathrm{MH}(\beta-\mathrm{HCH}, \mathrm{PCB} 180$, methoxychlor, anthracene) and AMDIS $(\beta-\mathrm{HCH})$.

Total average MF for all the substances in a standard mixture is 874 (near 900- "excellent match"), and for all the substances in the real samples, it is 786 (near 800- "good match") [25]. The calculated difference between the Total ARMFs and AMFs is 43 for standard mixture and 62 for the real samples. With the exception of a few compounds (e.g., acenaphtylene, pyrimethanil, anthracene, d-BHC, parathion-methyl, fenchlorphos, $p, p^{\prime}$-DDD, where the differences are higher), it can be concluded that these differences (due to background or co-eluting substance) are small and the deconvolution procedure was effective enough.

\section{Materials and Methods}

\subsection{Materials and Reagents}

Dichloromethane (DCM) and acetonitrile (ACN) were of pesticide residue grade from Merck (Poznań, Poland). Aluminium oxide 90 active neutral for column chromatography obtained from Merck (Poznań, Poland) was used as an SPE bed (200 mg).

\subsection{Preparation of Analytical Standards Solution}

Stock solutions of mixtures of pesticides, PCBs and PAHs, were obtained from Supelco (Poznań, Poland). Standard solutions were diluted from the stock solutions with dichloromethane. The concentration of each component in the standard solution was between 0.2 and $2 \mu \mathrm{g} / \mathrm{mL}$.

\subsection{Real Sample Preparation}

Two types of soil samples were collected. The total organic carbon (TOC) content was different, i.e., in the sample S1: $0.97 \%$ and in the sample S2: $4.41 \%$. The soil was dried at $40{ }^{\circ} \mathrm{C}$ and sieved (mesh size $0.43 \mathrm{~mm}$ ) at room temperature. Additionally, a sample of a high concentration of organic matter: cardboard (S3) used as a packaging material for oranges was also taken (cardboards are usually treated with various protective agents and contain post-production organic compounds).

The samples were prepared according to the procedure described earlier, with some modifications $[28,29]$. Soil samples $(3 \mathrm{~g})$ were extracted with DCM $(2 \times 8 \mathrm{~mL})$ in an ultrasonic bath for $20 \mathrm{~min}(2 \times 10 \mathrm{~min})$. The extract was evaporated under a gentle stream of nitrogen till dry. The dried residue was dissolved in $2 \times 300 \mu \mathrm{L}$ of ACN and cleaned up on alumina ( $200 \mathrm{mg}$ ) SPE bed. A fraction of $1 \mathrm{~mL}$ was eluted with acetonitrile, evaporated and dissolved in $300 \mu \mathrm{L}$ of standard solution $(2 \mu \mathrm{g} / \mathrm{mL})$. The samples were then analyzed using GC/MS.

\subsection{GC/MS Analysis}

The GC-MS analysis was performed using a 7890B gas chromatograph equipped with a 7693 autosampler and a 5977B mass-selective detector (Agilent, Santa Clara, CA, USA). The capillary column used was HP-5MS, $30 \mathrm{~m} \times 0.25 \mathrm{~mm} \times 0.25 \mathrm{~mm}$, from Agilent (Santa Clara, CA, USA). Helium was used as carrier gas at $1.5 \mathrm{~mL} / \mathrm{min}$. The split-splitless injector was operated in pulsed pressure splitless mode 
as follows: initial pressure $0.2 \mathrm{MPa}(30$ p.s.i.) for $1.3 \mathrm{~min}$, decreased to constant flow. The purge valve was opened after $1.5 \mathrm{~min}$. The injection volume was $5 \mu \mathrm{L}$. The temperatures of the GC system were the following: injector temperature $290^{\circ} \mathrm{C}$; transfer line temperature $280^{\circ} \mathrm{C}$; oven temperature program: $50{ }^{\circ} \mathrm{C}(1.5 \mathrm{~min})-30^{\circ} \mathrm{C} / \mathrm{min}-180^{\circ} \mathrm{C}-20^{\circ} \mathrm{C} / \mathrm{min}-280^{\circ} \mathrm{C}(20 \mathrm{~min})$. MS detector (quadrupole) was operated in the EI mode at $70 \mathrm{eV}$ with a mass scan range of $50-450 \mathrm{~m} / \mathrm{z}$ and the sampling rate of $3.6 \mathrm{scans} / \mathrm{s}$.

\subsection{Data Processing}

Several virtual machines with 12-20 processors (Intel Xeon E312xx or Intel Core i7 9xx, Santa Clara, CA, USA) and 16-32 GB RAM were used for Windows-based applications. All the software was operated with 64-bit Windows 8.1 Enterprise operating system.

\subsubsection{PARADISe Program}

PARADISe v 3.87 was used for the analysis standard solution, and real sample chromatograms obtained from GC/MS analysis. As mentioned earlier, it is rather uncommon-for environmental samples such as water or soil and sediments-to repeat analysis more than 2-3 times. This would make this software unusable due to few processed chromatograms. When analyzing the variability of the peaks (for one analyte) on GC chromatograms originated from one sample type (several repetitions), it can be observed that there are some differences among them in retention time and, in general, in peak shape (also caused by various concentrations). Such a variation is a necessary condition when using PARADISe software [11]. To artificially achieve such changes, several chromatograms were generated from a single GC/MS chromatogram after some mathematical modifications. The original file (acquired from GC/MS: standard mixtures and spiked extracts from the real samples) was modified with OpenChrom software [30] using multiplier filter and retention time shifter. Multiplier filter with the factors between 0.5 to 1.5 was used. The retention time shifter was applied with offset backwards or forward between 0 and $0.003 \mathrm{~min}$. These values were obtained by observing the retention time deviation of the analytes during the analysis of real samples. Several (usually four) additional chromatograms were created and exported to CDF files. This resulted in the minimum number of chromatograms (five) that were necessary for PARADISe to run. In the case of the real samples, an additional denoising filter with default settings was applied: this made it easier to establish an appropriate number of intervals (Figure S1). Subsequently, all chromatograms (one primary and four derived from it) were opened in PARADISe v 3.87 program and number of the intervals were established manually: for the standard mixture solution-it was about 60 and for the real samples from 93 to 107 (set individually for the sample of the same kind). The number of the intervals was higher than the number of compounds in the standard mixture, because for not fully separated substances, the peaks originating from them were selected in more than one interval: covering several compounds as well as only one, if possible. In this case, during the final analysis of the results, the same substance was discovered twice or more times (with the same retention time). This procedure was used due to the limitation of the PARADISe program: it cannot deconvolute peaks when the data interval contains substances with identical mass spectra-this is the case with not fully separated PAHs.

PARAFAC2 modelling was run with the following parameters: default number of components per interval: from 1 to 7; the maximum number of iterations: 50,000 and with Non-negativity field marked. On a twenty-processors (CPUs) virtual machine, the computation was completed in 2-3 h. Models were then evaluated according to the information provided in recently published articles $[11,21]$ and on the YouTube channel QualityAndTechnology [27], which provides step-by-step instructions on how to use this program and interpret the results obtained. Models for each interval were inspected individually. The number of components was established by analyzing all the information provided in the Model evaluation window to ensure adequate model fit (over 90\%), noise removal, and low residuals, with a core consistency over $90 \%$ (Figure 2). With the selected model for each interval, a report was generated (in xls file format) using NIST17 MS library. 


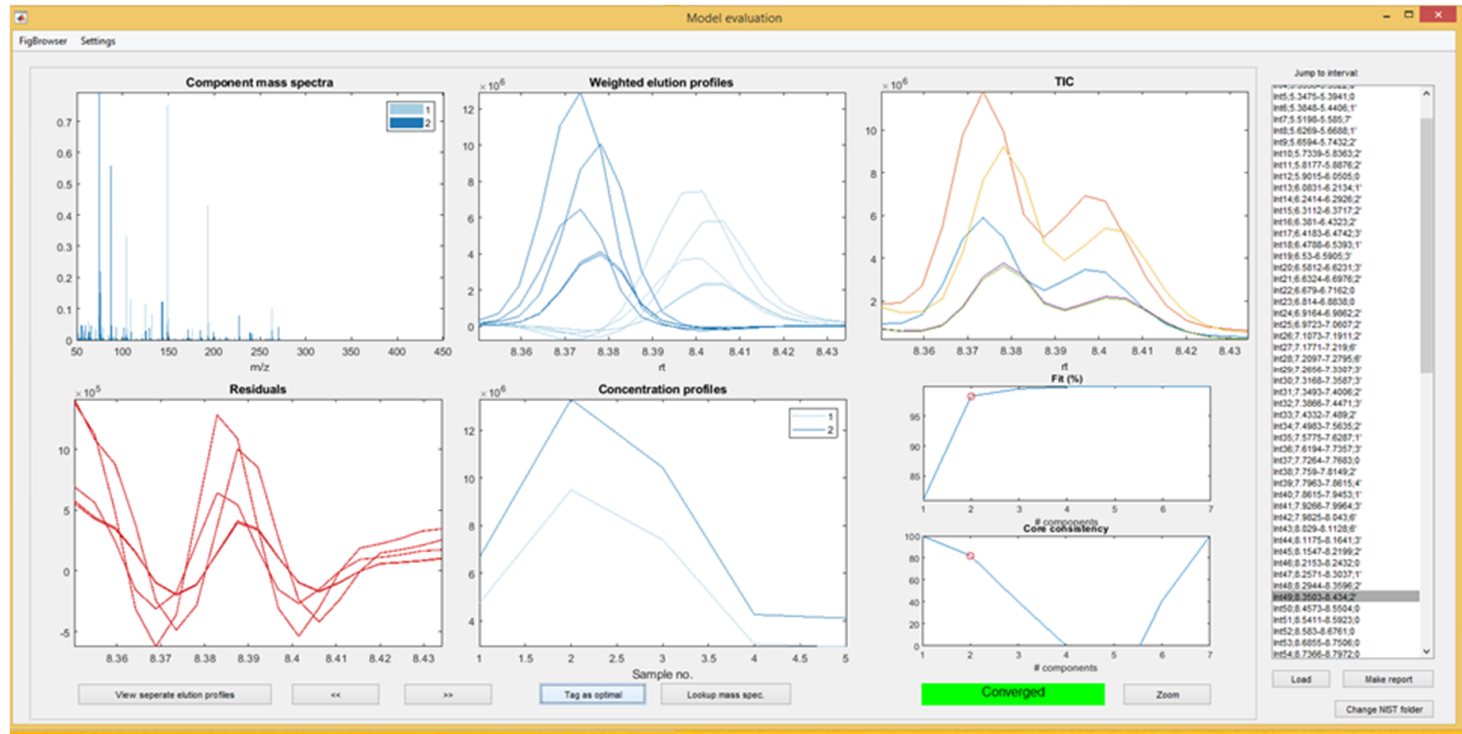

Figure 2. PARADISe model evaluation window with PCB 28 and parathion-methyl peaks (sample S3). Original chromatogram and four additional chromatograms derived from it. The original file was modified with the following parameters, which were generated randomly (retention time shifter [min], multiplier): (-0.00156, 1.99866); (0.00221, 1.56173); (0.00158, 0.63779); (-0.00107, 1.00608).

\subsubsection{AMDIS Program.}

For comparison, data analysis was also performed using the AMDIS program (version 2.73, NIST, Gaithersburg, MD, USA) together with the NIST17 MS library. For the AMDIS, the analysis type was used: Use Retention Index Data with target library NISTEPA.MSL. The following AMDIS deconvolution settings were used: Adjacent peak subtraction: One; Resolution: Medium; Sensitivity: Very Low; Shape requirements: Medium (similar to [3]). The Kovats indices were determined using the mixture of alkanes (C12-C27). AMDIS generated spectra for identified and unidentified compounds. A search of the NIST Library (NIST17) was then performed with a Minimum match factor of 75 for all components.

\subsubsection{MassHunter Program}

In addition, the GC/MS data were also evaluated with MassHunter (MH) Workstation Software Qualitative Analysis Workflows (version B.08.00, Agilent Technologies Inc., Santa Clara, CA, USA). It was operated with the following parameters: compounds were discovered by chromatogram deconvolution with the default settings; substances were identified using MS library (NIST17) search.

\section{Conclusions}

The proposed, simplified method, based on one chromatogram and limited data set generated from it can be efficiently used for the chromatogram deconvolution with the PARADISe software. The results obtained in the proposed procedure are comparable to those form AMDIS or MassHunter. PARADISe can be treated as complementary software to these two programs. Using the software tandem, like PARADISe and MH or PARADISe and AMDIS allows efficient qualitative analysis of the semivolatile pollutants (like PAHs, PCBs, organic pesticides) in the real samples. A high concentration of the matrix components (a common situation in the analysis of the environmental samples) leads to problems with the identification of the compound. In this case, it is necessary to clean up the sample enough to obtain a lower background (various methods, depending on the properties of the analytes, can be helpful for this purpose, mainly different types of solid phase extraction, adsorption chromatography, gel permeation chromatography, sulfuric acid cleanup, and other [28,31-33]), then the deconvolution can be an efficient tool in non-target analysis. 
Supplementary Materials: The following are available online at http://www.mdpi.com/1420-3049/25/16/3727/s1, Figure S1: Effect of denoising filter on chromatograms: five chromatograms (sample S3) imported as CDF files into PARADISe program A) without the denoising filter B) with the denoising filter applied earlier.

Funding: Financial support of the Rector of the UTP University of Science and Technology No. BN 10/2020.

Acknowledgments: The author is grateful to UTP University of Science and Technology for granting access to the computing infrastructure built in the projects No. POIG.02.03.00-00-028/08 "PLATON - Science Services Platform" and No. POIG.02.03.00-00-110/13 "Deploying high-availability, critical services in Metropolitan Area Networks (MAN-HA)".

Conflicts of Interest: The author declares no conflict of interest.

\section{References}

1. Dabrowski, Ł. Multidetector systems in gas chromatography. TrAC-Trends Anal. Chem. 2018, 102, $185-193$.

2. Dagan, S. Comparison of gas chromatography-pulsed flame photometric detection-mass spectrometry, automated mass spectral deconvolution and identification system and gas chromatography-tandem mass spectrometry as tools for trace level detection and identification. J. Chromatogr. A 2000, 868, 229-247. [CrossRef]

3. Lu, H.; Liang, Y.; Dunn, W.B.; Shen, H.; Kell, D.B. Comparative evaluation of software for deconvolution of metabolomics data based on GC-TOF-MS. TrAC-Trends Anal. Chem. 2008, 27, 215-227. [CrossRef]

4. Norli, H.R.; Christiansen, A.; Holen, B. Independent evaluation of a commercial deconvolution reporting software for gas chromatography mass spectrometry analysis of pesticide residues in fruits and vegetables. J. Chromatogr. A 2010, 1217, 2056-2064. [CrossRef] [PubMed]

5. Misra, B.B. New tools and resources in metabolomics: 2016-2017. Electrophoresis 2018, 39, 909-923. [CrossRef] [PubMed]

6. Bevilacqua, M.; Bro, R.; Marini, F.; Rinnan, Å.; Rasmussen, M.A.; Skov, T. Recent chemometrics advances for foodomics. Trac-Trends Anal. Chem. 2017, 96, 42-51. [CrossRef]

7. Amigo, J.M.; Skov, T.; Bro, R. ChroMATHography: Solving chromatographic issues with mathematical models and intuitive graphics. Chem. Rev. 2010, 110, 4582-4605. [CrossRef] [PubMed]

8. Cerdán-Calero, M.; Sendra, J.M.; Sentandreu, E. Gas chromatography coupled to mass spectrometry analysis of volatiles, sugars, organic acids and aminoacids in Valencia Late orange juice and reliability of the Automated Mass Spectral Deconvolution and Identification System for their automatic identifica. J. Chromatogr. A 2012, 1241, 84-95. [CrossRef]

9. An Integrated Method for Spectrum Extraction and Compound Identification from GC/MS Data. J. Am. Soc. Mass Spectrom. 1999, 10, 770-781. [CrossRef]

10. Behrends, V.; Tredwell, G.D.; Bundy, J.G. A software complement to AMDIS for processing GC-MS metabolomic data. Anal. Biochem. 2011, 415, 206-208. [CrossRef]

11. Johnsen, L.G.; Skou, P.B.; Khakimov, B.; Bro, R. Gas chromatography mass spectrometry data processing made easy. J. Chromatogr. A 2017, 1503, 57-64. [CrossRef] [PubMed]

12. Law, K.P.; Han, T.L.; Yang, Y.; Zhang, H. Analytical challenges of untargeted GC-MS-based metabolomics and the critical issues in selecting the data processing strategy. F1000Research 2017, 6, 967-978.

13. Risum, A.B.; Bro, R. Using deep learning to evaluate peaks in chromatographic data. Talanta 2019, 204, 255-260. [CrossRef] [PubMed]

14. Johnsen, L.G.; Amigo, J.M.; Skov, T.; Bro, R. Automated resolution of overlapping peaks in chromatographic data. J. Chemom. 2014, 28, 71-82. [CrossRef]

15. Kamstrup-Nielsen, M.H.; Johnsen, L.G.; Bro, R. Core consistency diagnostic in PARAFAC2. J. Chemom. 2013, 27, 99-105. [CrossRef]

16. Amigo, J.M.; Skov, T.; Bro, R.; Coello, J.; Maspoch, S. Solving GC-MS problems with PARAFAC2. Trac-Trends Anal. Chem. 2008, 27, 714-725. [CrossRef]

17. Tian, K.; Wu, L.; Min, S.; Bro, R. Geometric search: A new approach for fitting PARAFAC2 models on GC-MS data. Talanta 2018, 185, 378-386. [CrossRef]

18. Murphy, K.R.; Wenig, P.; Parcsi, G.; Skov, T.; Stuetz, R.M. Characterizing odorous emissions using new software for identifying peaks in chemometric models of gas chromatography-mass spectrometry datasets. Chemom. Intell. Lab. Syst. 2012, 118, 41-50. [CrossRef] 
19. Skou, P.B.; Khakimov, B.; Hansen, T.H.; Aunsbjerg, S.D.; Knøchel, S.; Thaysen, D.; van den Berg, F. Chemical characterization by gas chromatography-mass spectrometry and inductively coupled plasma-optical emission spectroscopy of membrane permeates from an industrial dairy ingredient production used as process water. J. Dairy Sci. 2018, 101, 135-146. [CrossRef]

20. Khakimov, B.; Mongi, R.J.; Sørensen, K.M.; Ndabikunze, B.K.; Chove, B.E.; Engelsen, S.B. A comprehensive and comparative GC-MS metabolomics study of non-volatiles in Tanzanian grown mango, pineapple, jackfruit, baobab and tamarind fruits. Food Chem. 2016, 213, 691-699. [CrossRef]

21. Sales, C.; Portolés, T.; Johnsen, L.G.; Danielsen, M.; Beltran, J. Olive oil quality classification and measurement of its organoleptic attributes by untargeted GC-MS and multivariate statistical-based approach. Food Chem. 2019, 271, 488-496. [CrossRef] [PubMed]

22. Zhang, Y.; Silcock, P.; Jones, J.R.; Eyres, G.T. Changes in wood smoke volatile composition by manipulating the smoke generation conditions. J. Anal. Appl. Pyrolysis 2020, 148, 104769. [CrossRef]

23. Toraman, H.E.; Abrahamsson, V.; Vanholme, R.; Van Acker, R.; Ronsse, F.; Pilate, G.; Boerjan, W.; Van Geem, K.M.; Marin, G.B. Application of Py-GC/MS coupled with PARAFAC2 and PLS-DA to study fast pyrolysis of genetically engineered poplars. J. Anal. Appl. Pyrolysis 2018, 129, 101-111. [CrossRef]

24. Alves Filho, E.G.; Silva, L.M.A.; Wurlitzer, N.J.; Fernandes, F.A.N.; Fonteles, T.V.; Rodrigues, S.; de Brito, E.S. An integrated analytical approach based on NMR, LC-MS and GC-MS to evaluate thermal and non-thermal processing of cashew apple juice. Food Chem. 2019, 309, 125761. [CrossRef] [PubMed]

25. NIST NIST mass spectral database for NIST/EPA/NIH and mass spectral search program (version 2.3). Natl. Inst. Stand. Technol. Nist 2017, 6, 1-73.

26. Dąbrowski, Ł.; Giergielewicz-Możajska, H.; Górski, Ł.; Biziuk, M.; Namieśnik, J.; Bogdan, J. Determination of environmental pollutants in soil and sediments-Some aspects of sample clean-up and GC analysis. J. Sep. Sci. 2002, 25, 290-296. [CrossRef]

27. Quality and Technology Channel. Available online: Youtube.com/user/QualityAndTechnology/videos. (accessed on 12 June 2020).

28. Dąbrowska, H.; Biziuk, M.; Gaca, J.; Namieśnik, J. Solid-phase extraction clean-up of soil and sediment extracts for the determination of various types of pollutants in a single run. J. Chromatogr. A 2003, 1003, $29-42$.

29. Dabrowski, Ł. Design and application of a gas chromatograph-gas chromatograph transfer line. J. Chromatogr. A 2008, 1210, 234-238. [CrossRef]

30. Wenig, P.; Odermatt, J. OpenChrom: A cross-platform open source software for the mass spectrometric analysis of chromatographic data. BMC Bioinform. 2010, 11, 405-411. [CrossRef]

31. Khan, Z.; Troquet, J.; Vachelard, C. Sample preparation and analytical techniques for determination of polyaromatic hydrocarbons in soils. Int. J. Environ. Sci. Technol. 2005, 2, 275-286. [CrossRef]

32. Muir, D.; Sverko, E. Analytical methods for PCBs and organochlorine pesticides in environmental monitoring and surveillance: A critical appraisal. Anal. Bioanal. Chem. 2006, 386, 769-789. [CrossRef] [PubMed]

33. Tadeo, J.L.; Pérez, R.A.; Albero, B.; García-Valcárcel, A.I.; Sánchez-Brunete, C. Review of Sample Preparation Techniques for the Analysis of Pesticide Residues in Soil. J. Aoac Int. 2012, 95, 1258-1270. [CrossRef] [PubMed]

(C) 2020 by the author. Licensee MDPI, Basel, Switzerland. This article is an open access article distributed under the terms and conditions of the Creative Commons Attribution (CC BY) license (http://creativecommons.org/licenses/by/4.0/). 